\title{
SELEÇÃO DE GENITORES DE Eucalyptus grandis E DE Eucalyptus urophylla PARA PRODUÇÃO DE HÍBRIDOS INTERESPECÍFICOS UTILIZANDO REML/ BLUP E INFORMAÇÃO DE DIVERGÊNCIA GENÉTICA ${ }^{1}$
}

\author{
Maria das Graças de Barros Rocha², Ismael Eleotério Pires ${ }^{3}$, Rodrigo Barros Rocha ${ }^{4}$, Aloísio Xavier ${ }^{3}$ e \\ Cosme Damião Cruz ${ }^{5}$
}

\begin{abstract}
RESUMO - Foram avaliadas 363 progênies de meios-irmãos de Eucalyptus urophylla e de 245 progênies de meios-irmãos de E. grandis, visando à seleção de genitores para a produção de híbridos interespecíficos em cruzamentos controlados em dialelo circulante, com base em seus valores genéticos preditos. Os ensaios foram conduzidos em Guanhães, MG, no delineamento de blocos casualizados com cinco repetições, parcelas lineares de seis plantas para E. urophylla e oito plantas para E. grandis, no espaçamento de 3,0 x 2,0 m. Os parâmetros genéticos foram estimados adotando-se modelos mistos com uso do procedimento REML/BLUP (máxima verossimilhança restrita/melhor predição linear não viesada) para diâmetro à altura do peito (DAP), altura total (ALT) e volume individual (VOL). A predição dos valores genéticos foi feita somente para (DAP), característica que foi utilizada para seleção. Em E. urophylla, foram obtidas as estimativas de herdabilidade no sentido restrito de 0,2785 e de acurácia da ordem de $61 \%$, e em E. grandis, herdabilidade de 0,2247 e acurácia de $53 \%$. A seleção dos 100 genitores de cada espécie individualmente proporcionará ganhos genéticos na ordem de $20,6 \%$ em $E$. urophylla e de $16,4 \%$ em E. grandis. Ganho genético para DAP em torno de 35,0\% poderá ser obtido com o cruzamento dos 10 indivíduos selecionados para cada espécie, portadores das maiores estimativas de divergência genética.
\end{abstract}

Palavras-chave: Melhoramento florestal, parâmetros genéticos e valor genético predito.

\section{SELECTION OF Eucalyptus grandis AND Eucalyptus urophylla PARENTS FOR THE PRODUCTION OF INTERSPECIFIC HYBRIDS USING REML/BLUP AND GENETIC DIVERSITY DATA}

\begin{abstract}
Predicted parental genetic values of 363 half-sib Eucalyptus grandis and 245 half-sib E. urophylla progenies were evaluated for selection to produce interspecific hybrids in controlled crosses in a circulating diallel. The essays were conducted in Guanhães, Minas Gerais, in a complete randomized block design in five replications, row plots of six E. urophylla and eight $\mathrm{E}$. grandis plants in $3.0 \times 2.0 \mathrm{~m}$ spacing. The genetic parameters were estimated by mixed models using the REML/BLUP (restricted maximum likelihood/best linear unbiased prediction) procedure for diameter at breast height (DBH), total height (TH) and individual volume (VOL) traits. The genetic values were only predicted for diameter at breast height (DBH), to which
\end{abstract}

\footnotetext{
${ }^{1}$ Recebido em 03.07.2006 e aceito para publicação em 24.05.2007.

${ }^{2}$ Instituto Estadual de Florestas - IEF. E-mail: <mgbrocha@yahoo.com.br>.

${ }^{3}$ Departamento de Engenharia Florestal da Universidade Federal de Viçosa -UFV. E-mail: <iepires@ufv.br>.

${ }^{4}$ EMBRAPA - Centro de Pesquisa Agroflorestal de Rondônia (CPAF-RO), BR 364, Km 5.5, 78900-000 Porto Velho,RO. E-mail: <rodrigo@cpafro.embrapa.br>.

${ }^{5}$ Departamento de Biologia Geral da UFV. E-mail: <cdcruz@ufv.br>.
} 
the selection was performed. The estimated heritability values in the restricted sense for Eucalyptus urophylla were 0.2785 and for accuracy $61 \%$, and 0.2247 and $53 \%$ in Eucalyptus grandis, respectively. Selection of 100 parents of each species individually will provide genetic gains of $20.6 \%$ in Eucalyptus urophylla and of $16.4 \%$ in Eucalyptus grandis. A genetic gain for diameter at breast height around $35.0 \%$ can be obtained by crosses among the first 10 selected individuals of each species and carriers of the greatest genetic divergences.

Keywords: Forest improvement, genetic parameters and mixed models.

\section{INTRODUÇÃO}

No Brasil, desde a década de 1970, os programas de melhoramento genético de eucalipto utilizam metodologias de hibridação e de clonagem como principais ferramentas para a obtenção de ganhos genéticos, fundamentados na expressiva divergência genética entre e dentro de espécies e procedências associada à expressiva manifestação heterótica dos híbridos (RESENDE e HIGA, 1990). Geralmente, observa-se superioridade dos híbridos interespecíficos de Eucalyptus spp., principalmente em relação às progênies parentais quanto ao crescimento, à adaptação e à tolerância a doenças.

No melhoramento genético de eucalipto, avanços foram conseguidos em termos de definição de espécies, procedências adaptadas aos ambientes de cultivo, adequação ao produto final, métodos de propagação, hibridação e silvicultura clonal, em que é possível programar os cruzamentos pela indução floral em genitores enxertados e conduzidos em casa de vegetação. Nesse cenário, é determinante para o sucesso do programa uma estratégia que tenha como fundamento a condução das hibridações, visando ao melhoramento do próprio híbrido ao longo das gerações com aumento continuado da heterose. A expectativa é de que os híbridos gerados entre os genitores com base em seus valores genéticos preditos em populações divergentes e, portanto, portadores de boa capacidade geral e específica de combinação apresentem superioridade ao longo das gerações em esquemas de seleção recorrente recíproca.

O êxito do melhoramento genético está associado à capacidade de acerto na escolha dos melhores indivíduos que serão os genitores das próximas gerações (CRUZ e CARNEIRO, 2003). Uma das maneiras de identificar os indivíduos portadores de genes desejáveis é a avaliação genética dos candidatos à seleção. Em plantas perenes, recomenda-se que a seleção seja feita com base nos valores genéticos aditivos dos indivíduos que serão utilizados na recombinação e nos valores genotípicos dos indivíduos que serão clonados (RESENDE, 2002a). O procedimento adequado para a predição dos valores genéticos utilizados na avaliação de plantas perenes tem sido o BLUP (melhor predição linear não viesada) individual que consiste, basicamente, na predição de valores genéticos dos efeitos aleatórios do modelo estatístico, associado às observações fenotípicas, ajustando-se os dados aos efeitos fixos e ao número desigual de informações nas parcelas, por meio de metodologia de modelos mistos (HENDERSON et al., 1959).

Considerando a seleção de híbridos interespecíficos superiores, é imprescindível a realização de cruzamentos controlados em um delineamento que prorize, tanto a capacidade geral (CGC) quanto a capacidade específica (CEC) de combinação. Quando o grupo de genitores é grande, vários obstáculos são encontrados, como a falta de sincronismo e o curto período de florescimento das espécies, grande quantidade de polinizações e necessidade de mão-de-obra especializada, associados ao alto custo da obtenção de sementes híbridas. No entanto, a análise de um número reduzido de cruzamentos, constituídos de apenas alguns genitores, faz que os estimadores das funções estimáveis dos efeitos da capacidade combinatória fiquem sujeitos a uma grande variação residual (CRUZ e PIRES, 1996).

Com a finalidade de contornar tais problemas, propõem-se o estudo da capacidade geral de combinação utilizando valores genéticos preditos pela metodologia de REML/BLUP e da capacidade específica de combinação utilizando informações de diversidade genética baseada em características silviculturais, visando à estruturação dos cruzamentos no esquema de dialelo parcial circulante. Nesse contexto, este trabalho teve como objetivo selecionar genitores de E. urophylla e de $E$. grandis portadores das maiores divergências 
genéticas, visando à produção de sementes melhoradas dessas espécies individualmente e à obtenção de híbridos interespecíficos em esquemas de cruzamentos de dialelo parcial circulante, utilizando métodos de seleção e de quantificação da diversidade genética.

\section{MATERIAL E MÉTODOS}

\subsection{Material experimental}

Utilizaram-se cinco testes de progênies de $E$. urophylla e um de E. grandis instalados em Guanhães, MG, latitude de $18^{\circ} 48^{\prime} 06^{\prime}$ 'S e longitude de $42^{\circ} 58^{\prime} 22^{\prime \prime} \mathrm{W}$. Os testes de E. urophylla foram instalados separadamente por procedência, TP-67 b1 (procedência Anhembi-SP; com 34 progênies); TP-68 b1 (procedência Alor-Indonésia, com 25 progênies); TP-69 b1 (procedência Mt. Lewotobi-Indonésia, com 60 progênies); TP-70 b1 (procedência Egon-Indonésia, com 118 progênies); e TP-71 b1 (procedência Wetar- Indonésia, com 126 progênies), totalizando 363 progênies, e o teste de $E$. grandis com 245 progênies envolveu 13 procedências da Austrália (240 progênies) e uma da CENIBRA (5 progênies). Os ensaios foram instalados em blocos casualizados com cinco repetições e parcelas lineares de oito e seis plantas, respectivamente. A avaliação foi feita aos 58 meses de idade, com medição de altura total (ALT), diâmetro à altura do peito (DAP) e volume individual (VOL) obtido com a fórmula:

$$
V O L U M E_{C C}=\frac{\pi(D A P)^{2} \cdot f f .}{40.000} A L T,
$$

em que fator de forma $f f=0,5$

\subsection{Estimativas dos parâmetros genéticos}

Os valores genéticos foram estimados por meio do procedimento REML/BLUP(Máxima Verossimilhança Restrita/Melhor Predição Linear Não Viesada) com relação às características DAP, ALT e VOL. A predição dos valores genéticos utilizando a metodologia de BLUP, a partir do sistema de equações de modelos mistos, pressume o conhecimento dos componentes de variância que são estimados utilizando o método de máxima verossimilhança restrita REML. Essa metodologia temse consolidado no setor florestal, principalmente por permitir a obtenção de estimativas mais acuradas de herdabilidade e de ganho de seleção provenientes da análise de experimentos desbalanceados.
Os parâmetros genéticos foram estimados pela análise conjunta dos cinco testes de progênies de $E$. urophylla e pela análise individual de teste de E. grandis. A predição de valores genéticos aditivos nos testes do E. urophylla foi obtida utilizando-se modelo linear misto, sem considerar os efeitos de procedências e, no teste de $E$. grandis, o modelo linear misto com várias procedências, conforme Resende (2000b).

A predição dos valores genéticos aditivos dos genitores foi feita utilizando-se o programa SelegenREML/BLUP, e o modelo linear misto univariado, conforme Resende (2002a):

$$
Y=X b+Z a+W c=e
$$

em que:

$Y=$ vetor de dados;

$X=$ matriz de incidência para o efeito fixo de bloco;

$b=$ vetor dos efeitos de bloco, tomado como fixo;

$Z=$ matriz de incidência do efeito aleatório de indivíduos;

$a=$ vetor dos efeitos genéticos aditivos tomados como aleatório;

$W=$ matriz de incidência para o efeito de parcela;

$c=$ vetor dos efeitos aleatórios de progênies; e $e=$ vetor de erros aleatórios.

As pressuposições acerca da distribuição de $y, a, c, e$ estruturas de médias e variâncias para cada vetor são dadas por:

$$
\begin{aligned}
& y \sim N(X b, V) ; a \sim N\left(0, A \sigma_{a}^{2}\right) ; c \sim N\left(0, I \sigma_{c}^{2}\right) ; \\
& \mathrm{e} \sim N\left(0, I \sigma_{e}^{2}\right) \\
& \quad \hat{\sigma}_{a}^{2}=\text { variância genética aditiva; } \\
& \quad \hat{\sigma}_{c}^{2}=\text { variância dentro da parcela }=(\text { ambiental dentro }
\end{aligned}
$$
de parcelas mais a genética não aditiva); e

$\hat{\sigma}_{e}^{2}=$ variância ambiental;

$\mathrm{Na}$ predição dos valores genéticos aditivos no teste de $E$. grandis, utilizou-se o modelo linear misto, considerando-se o modelo aditivo univariado com várias procedências, dado por:

$$
Y=X b+Z a+W c_{1}+U c_{2}+e
$$

R. Árvore, Viçosa-MG, v.31, n.6, p.977-987, 2007 
em que:

$Y=$ vetor de dados;

$X=$ matriz de incidência para o efeito fixo de bloco;

$b=$ vetor dos efeitos de bloco, tomado como fixo;

$Z=$ matriz de incidência do efeito aleatório de indivíduos;

$a=$ vetor dos efeitos genéticos aditivos tomados como aleatório;

$W=$ matriz de incidência do afeito de parcela;

$c_{1}=$ vetor dos efeitos aleatórios de parcelas referentes a progênies;

$U=$ matriz de incidência dos valores genéticos de procedências;

$c_{2}=$ vetor dos efeitos aleatórios de parcelas referentes a procedências; e

$e=$ vetor de erros aleatórios

As pressuposições acerca da distribuição de $y, a, c_{l}, c_{2}, e$ estruturas de médias e variâncias de cada vetor são dadas por:

$Y \sim N(X b, V) ; a \sim N\left(0, A \sigma_{a}^{2}\right) ; c_{1} \sim N\left(0, I \sigma_{c_{1}}^{2}\right)$;

$; c_{2} \sim N\left(0, I \sigma_{c_{2}}^{2}\right) ; \mathrm{e} \sim N\left(0, I \sigma_{e}^{2}\right)$

$\sigma_{a}^{2}=$ variância genética aditiva;

$\sigma_{c_{l}}^{2}=$ variância fenotípica dentro de parcelas;

$\sigma_{c_{2}}^{2}=$ variância fenotípica dentro de procedências; e

$\sigma_{e}^{2}=$ variância ambiental.

\subsection{Divergência genética}

Para obtenção da divergência genética, foram estimadas as distâncias euclidianas médias a partir dos valores individuais de DAP, ALT e VOL (CRUZ e REGAZZI, 1994), utilizando-se o programa GENES (Programa Computacional em Genética e Estatística) (CRUZ, 1997), sendo:

$$
\left.d_{i i^{\prime}}=\sqrt{\frac{1}{n} \sum\left(X_{i j}\right.}-X_{i^{\prime} j}\right)^{2}
$$

em que $X_{i j}$ é a observação do i-ésimo progenitor $(\mathrm{i}=1,2, \ldots 200)$ e da $\mathrm{j}$ - ésima característica $(\mathrm{j}=1,2,3)$.

O agrupamento dos genitores foi feito pelo método de Tocher, citado por Cruz e Regazzi (1994), também com uso do programa GENES.

R. Árvore, Viçosa-MG, v.31, n.6, p.977-987, 2007

\subsection{Dialelo circulante}

O algoritmo para o estabelecimento dos cruzamentos no dialelo parcial circulante teve como orientação o trabalho de Cruz e Pires (1996), com base nos valores de Kemphorne e Curnow (1961), como segue:

Número de cruzamentos $=\frac{p . s}{2}$

em que:

p: número de progenitores a serem estudados; e

$s$ : número de combinações híbridas de cada progenitor, sendo $s<p-1$ e $s \geq 3$ para dialelos que incluem apenas a geração $\mathrm{F}_{1}$.

\subsection{Ganho genético}

Na estimativa de ganho nos cruzamentos híbridos, utilizaram-se duas expressões: na primeira, foi utilizada a heterose média de $15 \%$ para o cruzamento de $E$. urophylla $\mathrm{x} E$. grandis e os efeitos genéticos aditivos médios obtidos nas populações analisadas individualmente $\frac{1}{2}\left(\hat{g}_{\mu}+\hat{g}_{g}\right)$, supondo correlação com os efeitos genéticos aditivos interpopulacionais:

$$
\hat{G}_{(\mu g)}=\frac{1}{2}\left(\hat{\mu}_{u}+\hat{\mu}_{g}\right)+\hat{h}_{(\mu g)}+\frac{1}{2}(\hat{g} \mu+\hat{g} g)
$$

em que:

$\hat{G}(\mu g)=$ valor genotípico de um híbrido;

$\hat{\mu}_{u} e \hat{\mu}_{g}=$ médias gerais das populações selecionadas;

$\hat{h}_{(u g)}=$ heterose de E. urophylla $\times$ E. grandis (15\%); e

$(\hat{g} \mu$ e $\hat{g} g)=$ efeitos genéticos aditivos dos genitores selecionados E. urophylla x E. grandis.

Na segunda (CRUZ e CARNEIRO, 2003), foram utilizados os maiores valores de divergência genética entre os pares de cruzamentos, em substituição ao valor da heterose média.

$$
\hat{G}_{(\mu g)}=\frac{1}{2}\left(\hat{\mu}_{u}+\hat{\mu}_{g}\right)+d_{u g}+\frac{1}{2}\left(\hat{g}_{u}+\hat{g}_{g}\right)
$$

em que:

$\hat{G}(\mu g)=$ valor genotípico de um híbrido;

$\hat{\mu}_{u} e \hat{\mu}_{g}=$ médias gerais das populações selecionadas; 
$\hat{d}_{\text {ug }}=$ divergência genética entre os genitores; e

$\left(\hat{g}_{u} e \hat{g}_{g}\right)=$ efeitos genéticos aditivos dos genitores selecionados E. urophylla $\times$ E. grandis.

\section{RESULTADOS E DISCUSSÃO}

\subsection{Estimativas das variâncias, coeficientes de variação e herdabilidade}

Conforme se verifica no Quadro 1, os coeficientes de variação experimental médios de DAP $(7,43 \%)$, $\operatorname{ALT}(6,43 \%)$ e $\operatorname{VOL}(28,27 \%)$ ficaram dentro dos padrões normalmente encontrados em Eucalyptus (GARCIA, 1989), indicando boa precisão na análise dos dados. As médias e variâncias dos caracteres em estudo também estão dentro dos padrões das espécies estudadas e idade avaliada, e o E. grandis demonstrou maior produtividade no local de cultivo (Quadro 1). A análise de variância indicou a existência de variabilidade genética entre as progênies dos três caracteres avaliados a $1 \%$ de probabilidade, condição essa necessária para a obtenção de ganhos com a seleção.

Na identificação e seleção dos genótipos superiores, a variância genética aditiva se caracteriza como uma das ferramentas mais importantes para a quantificação do potencial de melhoramento da população e para o mérito da estratégia de seleção utilizada, visando à alteração da frequiência dos alelos favoráveis (CRUZ e REGAZZI, 1994). As estimativas da variância genética aditiva e do quociente entre a variância genética aditiva e a variância total (Quadro 1) sugerem significativa natureza aditiva no padrão de herança complexa dos caracteres avaliados, o que proporciona uma condição bastante favorável para a obtenção de ganhos com a prática da seleção.

O coeficiente de variação genética aditiva (CVa) é outro parâmetro usualmente utilizado para comparar a variabilidade genética expressa para cada caráter avaliado, conforme destacado por Resende (2001). Os coeficientes de variação de DAP e ALT foram similares nas duas espécies e o de VOL foi maior em E. urophylla (Quadro 2). Valores elevados de coeficiente de variação para VOL também foram encontrados por Pires (1996), em E. camaldulensis, que se justifica pelo fato de ser um caráter derivado e dependente das mensurações de ALT e DAP e, ainda, considerando erros de mensuração do caráter ALT associado, principalmente, às áreas declivosas, como é o caso, deve ter contribuído para a maior variação ambiental observada nos caracteres ALT e VOL.

Quadro 1 - Estimativas da variância genética aditiva $\left(\hat{\sigma}_{a}^{2}\right)$, variância dentro de parcelas $\left(\hat{\sigma}_{c}^{2}\right)$, variância ambiental entre parcelas $\left(\hat{\sigma}_{e}^{2}\right)$, variância fenotípica entre plantas no bloco $\left(\hat{\sigma}_{F}^{2}\right)$, coeficiente de variação experimental $C V_{\exp }$ (\%) e média dos caracteres diâmetro à altura do peito (DAP), altura total (ALT) e volume (VOL) em progênies de meios-irmãos de Eucalyptus urophylla e de Eucalyptus grandis, aos 58 meses de idade

Table 1 -Additive genetic variance $\left(\hat{\sigma}_{a}^{2}\right)$, environmental variance between plots $\left(\hat{\sigma}_{c}^{2}\right)$, phenotypic variance between plants within progenies $\left(\hat{\sigma}_{e}^{2}\right)$, phenotypic variance between plants within blocks $\left(\hat{\sigma}_{F}^{2}\right)$, variation coefficient $C V_{\text {exp }}(\%)$, and means of traits DBH, TH and VOL of half sib progenies of E. grandis and E. urophylla in field tests, at 58 months of age

\begin{tabular}{lcccccccc}
\hline \multirow{2}{*}{ Espécie } & Características & Média & CV exp & $\hat{\sigma}_{a}^{2}$ & $\hat{\sigma}_{c}^{2}$ & $\hat{\sigma}_{e}^{2}$ & $\hat{\sigma}_{F}^{2}$ & $\frac{\hat{\sigma}_{a}^{2}}{\hat{\sigma}_{F}^{2}}$ \\
\hline E.urophylla & DAP (cm) & 11,90 & 5,06 & 2,826 & 0,3630 & 5,5502 & 8,1957 & 0,35 \\
& ALT (m) & 16,96 & 4,76 & 2,6365 & 0,6518 & 3,8885 & 7,1768 & 0,37 \\
& VOL (m $\left.{ }^{3} \cdot \operatorname{arv}-1\right)$ & 0,0943 & 32,92 & 0,000967 & 0,000967 & 0,000967 & 0,00967 & 0,10 \\
\hline E.grandis & DAP (cm) & 11,91 & 9,81 & 2,2314 & 0,4574 & 7,1393 & 9,9306 & 0,23 \\
& ALT (m) & 18,09 & 8,11 & 2,4687 & 1,2799 & 5,0464 & 8,8972 & 0,28 \\
& VOL (m $\left.{ }^{3} \cdot \operatorname{arv}-1\right)$ & 0,1008 & 23,62 & 0,0006 & 0,0002 & 0,0002 & 0,003 & 0,2 \\
\hline
\end{tabular}


Quadro 2 - Estimativas do coeficiente de variação genética aditiva $C V_{\mathrm{a}}(\%)$, do coeficiente de variação dentro de parcelas $C V(\%)$, do coeficiente de variação ambiental entre parcelas $C V_{\mathrm{e}}(\%)$ e do coeficiente de variação fenotípica $C V_{\mathrm{F}}(\%)$ das características diâmetro à altura do peito (DAP), altura total (ALT) e volume (VOL) em progênies de meiosirmãos de Eucalyptus urophylla e de Eucalyptus grandis, aos 58 meses de idade

Table 2 -Additive genetic variation coefficient $C V_{a}(\%)$, variation coefficient inside plots $C V_{c}(\%)$, variation coefficient among plots $C V_{e}(\%)$, phenotypic variation coefficient $C V_{F}(\%)$ for traits $D B H, T H$ and VOL of half sib progenies of E. grandis and E. urophylla in field tests, at 58 months of age

\begin{tabular}{lccccc}
\hline Espécie & Caracteres & $C V_{\mathrm{a}}$ & $C V_{\mathrm{c}}$ & $C V_{\mathrm{e}}$ & $C V_{\mathrm{F}}$ \\
\hline E. urophylla & DAP $(\mathrm{cm})$ & 12,68 & 5,05 & 19,78 & 24,03 \\
& ALT (m) & 9,57 & 4,76 & 11,63 & 15,80 \\
& $\operatorname{VOL~}\left(\mathrm{m}^{3} \cdot \operatorname{arv}^{-1}\right)$ & 32,97 & 12,68 & 47,49 & 59,20 \\
\hline E. grandis & DAP $(\mathrm{cm})$ & 12,54 & 5,68 & 22,43 & 26,45 \\
& ALT (m) & 8,89 & 6,25 & 12,41 & 16,48 \\
& VOL $\left(\mathrm{m}^{3} \cdot \operatorname{arv}^{-1}\right)$ & 24,30 & 14,03 & 14,37 & 16,89 \\
\hline
\end{tabular}

O coeficiente de determinação devido ao ambiente comum da parcela quantifica a variabilidade dentro dos blocos, em que o alto valor desse coeficiente significa alta variabilidade ambiental dentro das parcelas. Em experimentos considerados precisos para plantas perenes, os valores dessa correlação devem estar em torno de 0,10 (RESENDE, 2002b). Somente para o caráter VOL esse valor foi superior a 0,10 (Quadro 3). O desviopadrão da herdabilidade (FALCONER, 1987) também pode ser utilizado como uma medida de qualidade para ponderar a adequação do uso dessas estimativas para predição dos valores genéticos. Segundo Resende (2002b), valores de desvio-padrão até $20 \%$ do valor da estimativa da herdabilidade indica boa precisão dessas estimativas. Nesse caso, somente a característica VOL apresentou valores de desvio-padrão superiores a essa referência (0,25 E. urophylla, 0,29 E. grandis).

As estimativas de herdabilidades, no sentido restrito em nível de plantas também indicaram a existência de considerável variabilidade genética nessas populações e considerando que a herdabilidade, no sentido restrito, tem a finalidade de orientar o melhorista sobre a quantidade relativa de variância genética útil para o melhoramento em descendências propagadas sexuadamente (FALCONER, 1987). Os valores dessas estimativas reforçam as boas perspectivas em relação ao avanço genético dos caracteres avaliados
(Quadro 3). As herdabilidades estimadas para os caracteres de crescimento variaram entre 0,15 e 0,50 , em nível de idivíduos, sendo coerentes com os valores citados na literatura para tais características (MORAES, 1987; PIRES, 1996; SAMPAIO et al., 2002; GARCIA e NOGUEIRA, 2005).

Em relação ao caráter DAP e considerado mais importante para fins de seleção para produção, foram obtidas as estimativas de herdabilidade, no sentido restrito, de 0,2785 e acurácia da ordem de $61 \%$ para E. urophylla e herdabilidade de 0,2247 e acurácia de $53 \%$ para E. grandis, valores esses considerados satisfatórios para a seleção em DAP, uma vez que outros trabalhos têm mostrado que a herdabilidade para essa característica em Eucalyptus está próximo a 0,30 (PIRES, 1996; MARTINS et al., 2003).

\subsection{Estimativas dos ganhos de seleção}

Optou-se por conduzir a seleção, utilizando o caráter DAP, devido à sua facilidade de avaliação e precisão, além de apresentar alta correlação, genética aditiva com ALT e VOL (MARTINS et al., 2003; SAMPAIO et al., 2002), visando à obtenção de ganhos genéticos expressivos também para volume. Foram selecionados 100 indivíduos de cada espécie de maior valor genético aditivo predito pelo REML/BLUP, para serem cruzados em esquema de dialelo circulante.

Quadro 3 - Estimativas dos coeficientes de herdabilidade no sentido restrito em nível de indivíduo $\left(h_{a}^{2}\right)$, de média de progênies $\left(\hat{h}_{m p}^{2}\right)$, dos coeficientes de determinação devido ao ambiente comum da parcela $\left(c_{p}^{2}\right)$ dos caracteres diâmetro à altura do peito (DAP), altura total (ALT) e volume (VOL) em progênies de meios-irmãos de Eucalyptus urophylla e de Eucalyptus grandis, aos 58 meses de idade

Table 3 -Restrict individual heritability coefficient estimates $\left(h_{a}^{2}\right)$ and family means $\left(\hat{h}_{m p}^{2}\right)$, determination coefficient of the plot effects $\left(c_{p}^{2}\right)$ for traits $D B H, T H$ and $V O L$ of half sib progenies of E. grandis and E. urophylla in field tests, at 58 months of age

\begin{tabular}{lcccc}
\hline Espécie & Caracteres & $h_{a}^{2}$ & $\hat{h}_{m p}^{2}$ & $c_{p}^{2}$ \\
\hline \multirow{3}{*}{ E.urophylla } & DAP $(\mathrm{cm})$ & 0,2785 & 0,9182 & 0,0444 \\
& ALT $(\mathrm{m})$ & 0,3674 & 0,9225 & 0,0908 \\
& $\operatorname{VOL~}\left(\mathrm{m}^{3} \cdot \mathrm{arv}^{-1}\right)$ & 0,3104 & 0,9258 & 0,0446 \\
\hline \multirow{2}{*}{ E.grandis } & DAP $(\mathrm{cm})$ & 0,2247 & - & 0,0461 \\
& ALT (m) & 0,2778 & - & 0,1438 \\
& VOL $\left(\mathrm{m}^{3} \cdot\right.$ arv $\left.^{-1}\right)$ & 0,2111 & - & 0,0558 \\
\hline
\end{tabular}


A seleção para DAP proporcionou estimativas de ganhos de $20,60 \%$ e de $15,44 \%$ para E. urophylla $\mathrm{e}$ E. grandis, respectivamente, e ganhos indiretos na ordem de 53,00\% e 29,76\% em VOL individual. Elevandose, assim, a média de DAP da população em um único ciclo de seleção de 11,90 para 14,36 para $E$. urophylla e de 11,91 para 13,75 para $E$. grandis e a média de VOL de 0,0943 para 0,1443 para E. urophylla e de 0,1008 para 0,1308 para $E$. grandis, conforme mostrado no Quadro 4. A predominância dos efeitos aditivos na herança do DAP de E. urohylla em relação a E. grandis resultou em estimativas de ganhos de seleção superiores; no entanto, essa diferença não apresentou magnitude suficiente para sugerir controle genético diferenciado desse caráter entre as duas espécies.

Considerando que os valores fenotípicos são diretamente medidos, mas que são os valores genéticos aditivos os responsáveis pela transmissão do caráter às próximas gerações (FALCONER, 1987) e que a acurácia corresponde à correlação entre os valores genéticos preditos e os verdadeiros (VAN VLECK et al.,1987), então, quanto maior for essa correlação, maior será a confiança na predição do valor genético. As estimativas da acurácia encontrados na predição dos valores genéticos pelo método REML/BLUP, apresentadas no Quadro 4, estão de acordo com os valores citados por Resende et al. (1990), na seleção de Pinus caribaea e de Mimosa scabrella, respectivamente.

No estabelecimento do pomar de sementes clonal (PSC), deve-se considerar que o aumento no número de indivíduos selecionados faz que o ganho genético diminua em conseqüência da redução da intensidade de seleção (Quadro 5). Resende (2002b) sugeriu que o número ideal de indivíduos selecionados deve ser definido com a maximização do limite inferior do intervalo de confiança do ganho genético corrigido para a endogamia. No estabelecimento das populações de produção de sementes, devem ser utilizadas as informações dos ganhos genéticos corrigidos para a endogamia (RESENDE e BERTOLUCCI, 1995) e sobre os intervalos de confiança para assegurar um número adequado de indivíduos na recombinação.

A maximização do limite inferior do intervalo de confiança do ganho genético corrigido para a endogamia em E. urophylla ocorreu com a seleção dos 20 indivíduos de maior valor genético, com um coeficiente de endogamia potencial de $2,6 \%$ e, em E. grandis, com a seleção de 30 indivíduos, com um coeficiente de endogamia potencial de 2,2\%. A seleção desses indivíduos proporcionou uma estimativa de ganho genético em DAP da ordem de $30,31 \%$ no E. urophylla e de $23,91 \%$ no E. grandis (Quadro 5). Os expressivos ganhos estimados se justificam pela existência de variabilidade genética expressa pelos respectivos coeficientes de herdabilidade.

\subsection{Divergência genética}

O agrupamento dos indivíduos, utilizando o método de otimização de Tocher (CRUZ e REGAZZI, 1994), resultou na separação dos 100 genitores em quatro grupos. Esse método se baseia em um valor prédeterminado $\theta$ como critério para inclusão ou não de um indivíduo a um grupo. Esse valor $\theta$, limite para inclusão no grupo, é influenciado pela presença de valores discrepantes, o que, geralmente, resulta no agrupamento dos genótipos em poucos grupos. Nesse caso, recomendam-se novas tentativas de agrupamentos, omitindo-se os genótipos mais discrepantes. Utilizandose essa estratégia, o agrupamento dos genótipos alocados apenas no grupo 1 resultou na obtenção de 26 novos grupos (Quadro 6). Embora tenham sido obervados grupos constituídos por indivíduos das espécies de E. urophylla e E. grandis, observa-se uma tendência de divergência entre essas espécies em relação aos caracteres avaliados.

Quadro 4 - Valores genéticos aditivos preditos, ganho genético dos caracteres altura total (ALT) e volume (VOL) e acurácia do caráter diâmetro à altura do peito (DAP) na seleção dos genitores em progênies de meios-irmãos de Eucalyptus urophylla e de Eucalyptus grandis, aos 58 meses de idade

Table 4 - Predicted additive genetic values, genetic gains for traits TH and VOL and selection accuracy of DBH for half sib progenies of E. grandis and E. urophylla in field tests at 58 months of age

\begin{tabular}{|c|c|c|c|c|c|c|}
\hline \multirow[t]{2}{*}{ Parâmetros } & \multicolumn{3}{|c|}{ Eucalyptus urophylla } & \multicolumn{3}{|c|}{ Eucalyptus grandis } \\
\hline & $\mathrm{DAP}(\mathrm{cm})$ & $\operatorname{ALT}(\mathrm{m})$ & $\operatorname{VOL}\left(\mathrm{m}^{3} \cdot \operatorname{arv}^{-1}\right)$ & DAP $(\mathrm{cm})$ & $\operatorname{ALT}(\mathrm{m})$ & $\operatorname{VOL}\left(\mathrm{m}^{3} \cdot \operatorname{arv}^{-1}\right)$ \\
\hline Valor genético predito & 14,36 & 18,50 & 0,1443 & 13,75 & 19,52 & 0,1008 \\
\hline Ganho & 2,45 & 1,54 & 0,05 & 1,84 & 1,43 & 0,03 \\
\hline Ganho (\%) & 20,60 & 9,08 & 53,00 & 15,44 & 7,90 & 29,76 \\
\hline Acurácia & 0,61 & - & - & 0,53 & - & - \\
\hline
\end{tabular}


Quadro 5 - Resultados referentes ao estabelecimento do pomar de sementes clonal - PSC de E. urophylla e de E. grandis, considerando-se diferentes números de indivíduos selecionados

Table 5 - Establishment of E. urophylla and E. grandis PSC population considering different numbers of selected individuals

\begin{tabular}{|c|c|c|c|c|c|c|c|c|c|c|}
\hline \multirow[b]{2}{*}{$\mathrm{n}$} & \multicolumn{5}{|c|}{ Eucalyptus urophylla } & \multicolumn{5}{|c|}{ Eucalyptus grandis } \\
\hline & $\mathrm{N}_{e}$ & $\mathrm{~F}$ & $\mathrm{G}$ & $\mathrm{G}(\%)$ & IC $(\%)$ & $\mathrm{N}_{e}$ & $\mathrm{~F}$ & $\mathrm{G}$ & $\mathrm{G}(\%)$ & IC (\%) \\
\hline 3 & 3,1 & 0,161 & 3,07 & 25,77 & $11,48 \_40,17$ & 2,4 & 0,208 & 3,39 & 21,78 & $12,96 \_444,26$ \\
\hline 10 & 9,1 & 0,055 & 2,97 & 24,96 & $16,71 \_33,46$ & 6,5 & 0,077 & 2,56 & 20,32 & $11,82 \_31,16$ \\
\hline 20 & 19,1 & 0,026 & 2,93 & 24,56 & $18,95 \_30,31$ & 11,8 & 0,042 & 2,28 & 19,40 & $11,98 \_21,30$ \\
\hline 30 & 25,1 & 0,020 & 2,85 & 23,90 & $18,92 \_28,91$ & 21,7 & 0,023 & 2,22 & 18,89 & $13,37 \_23,91$ \\
\hline 50 & 39,4 & 0,013 & 2,59 & 21,70 & $17,80_{-} 25,72$ & 30,5 & 0,016 & 2,12 & 18,04 & $13,33 \_22,25$ \\
\hline 100 & 70,0 & 0,007 & 2,45 & 20,60 & $17,61 \_23,56$ & 58,1 & 0,008 & 1,96 & 15,44 & $13,23 \_19,68$ \\
\hline
\end{tabular}

n: número de indivíduos selecionados; $\mathrm{N}_{e}$ : tamanho efetivo populacional; F: coeficiente de endogamia; G: ganho genético e IC: intervalo de confiança a $95 \%$ de probabilidade.

Quadro 6 - Agrupamento dos genitores de E. urophylla e Eucalyptus grandis pelo método de otimização de Tocher, baseado na dissimilaridade expressa pela distância euclidiana média $\left(\mathrm{d}_{\text {... }}\right)$

Table 6 -Clustering of the genitors of E. urophylla and E. grandis by the Tocher optimization method, based on Euclidian distances $\left(d_{i i}\right)$

\begin{tabular}{|c|c|c|c|c|c|c|c|c|c|c|c|c|c|c|c|}
\hline \multirow{2}{*}{$\frac{\text { Grupos }}{<1.1>}$} & \multicolumn{15}{|c|}{ Genitores } \\
\hline & $\mathrm{g} 4$ & g36 & g61 & g5 & g27 & g9 & g22 & g73 & $\mathrm{g} 80$ & g53 & g69 & g81 & g14 & g97 & g98 \\
\hline & g44 & g56 & g57 & g93 & g76 & g88 & g89 & g91 & u 138 & u 189 & $\mathrm{~g} 16$ & g30 & g72 & g67 & $\mathrm{g} 11$ \\
\hline & g99 & g42 & u 180 & g64 & g71 & g84 & & & & & & & & & \\
\hline$<1.2>$ & g8 & g39 & $\mathrm{g} 41$ & g46 & g47 & g82 & g18 & g68 & g13 & g52 & u 114 & g3 & g59 & g77 & g85 \\
\hline & g87 & g50 & u 150 & $\mathrm{~g} 25$ & g45 & u 164 & g21 & u 118 & u 108 & g38 & & & & & \\
\hline$<1.3>$ & g10 & g86 & g 100 & g63 & g55 & g1 & g75 & g7 & & & & & & & \\
\hline$<1.4>$ & $\begin{array}{c}\mathrm{g} 24 \\
\mathrm{u} 193\end{array}$ & g70 & g54 & g43 & u 160 & g31 & u 187 & g34 & u 159 & u 188 & u 128 & u 139 & u 158 & u 165 & g83 \\
\hline$<1.5>$ & $\mathrm{g} 28$ & g66 & g74 & g12 & u 192 & g6 & u 147 & u 176 & u 156 & u 123 & u 144 & g23 & u 132 & u 119 & u 154 \\
\hline & u 155 & u 107 & u 182 & u 178 & u 136 & u 137 & u 146 & u 135 & & & & & & & \\
\hline$<1.6>$ & g32 & g49 & g95 & g90 & g96 & g94 & g65 & u 184 & & & & & & & \\
\hline$<1.7>$ & u 173 & u 195 & g60 & $\mathrm{g} 40$ & u 191 & u 163 & u 152 & g58 & u 134 & u 149 & u 179 & u 197 & u 169 & u 183 & u 145 \\
\hline$<1.8>$ & g51 & u 121 & u 111 & u 168 & g17 & u 127 & & & & & & & & & \\
\hline$<1.9>$ & u 124 & u 177 & u 181 & u 171 & u 133 & u 186 & u 125 & u 140 & u 115 & u 141 & u 166 & u 161 & & & \\
\hline$<1.10>$ & g26 & g35 & g2 & g92 & g20 & g33 & g29 & g48 & u 199 & $\mathrm{u} 200$ & u 175 & & & & \\
\hline$<1.11>$ & u 174 & u 190 & u 151 & u 148 & u 122 & u 196 & u 120 & u 112 & & & & & & & \\
\hline$<1.12>$ & g62 & g79 & g37 & & & & & & & & & & & & \\
\hline$<1.13>$ & u 117 & u 143 & u 113 & u 131 & & & & & & & & & & & \\
\hline$<1.14>$ & u 162 & u 167 & u 110 & u 116 & & & & & & & & & & & \\
\hline$<1.15>$ & u 153 & u 172 & & & & & & & & & & & & & \\
\hline$<1.16>$ & u 109 & u 142 & & & & & & & & & & & & & \\
\hline$<1.17>$ & u 129 & & & & & & & & & & & & & & \\
\hline$<1.18>$ & u 126 & & & & & & & & & & & & & & \\
\hline$<1.19>$ & u 130 & & & & & & & & & & & & & & \\
\hline$<1.20>$ & u 104 & & & & & & & & & & & & & & \\
\hline$<1.21>$ & u 157 & & & & & & & & & & & & & & \\
\hline$<1.22>$ & u 194 & & & & & & & & & & & & & & \\
\hline$<1.23>$ & g78 & & & & & & & & & & & & & & \\
\hline$<1.24>$ & u 170 & & & & & & & & & & & & & & \\
\hline$<1.25>$ & u 185 & & & & & & & & & & & & & & \\
\hline$<1.26>$ & g19 & & & & & & & & & & & & & & \\
\hline$<2>$ & u 102 & u 103 & u 101 & u 106 & & & & & & & & & & & \\
\hline$<3>$ & g15 & u 105 & & & & & & & & & & & & & \\
\hline$<4>$ & u 198 & & & & & & & & & & & & & & \\
\hline
\end{tabular}

$\mathrm{u}-$ E. urophylla; $\mathrm{g}-$ E. grandis

R. Árvore, Viçosa-MG, v.31, n.6, p.977-987, 2007 
Além dos caracteres silviculturais, naturalmente contrastantes entre essas duas espécies, a manutenção da identidade genética das populações "espécie-pura" ao longo dos ciclos de seleção é uma particularidade do processo de melhoramento que também pode ter contribuído para uma maior distinção entre as espécies. Tão importante quanto à manutenção da identidade genética das populações, a identificação de conjuntos de genitores de maior capacidade específica de combinação e a conservação de base genética ampla são de grande importância para o programa florestal que se baseiam principalmente na produção de híbridos interespecíficos.

Devido à sua facilidade de interpretação, o método de otimização de Tocher é indicado para avaliação da divergência genética entre grande número de acessos, em função dos agrupamentos mutuamente exclusivos e não vazios, dados por esse método. A seleção dos genitores mais divergentes a partir de um grande conjunto inicial, visando à definição dos cruzamentos dialélicos, mostrou-se de interpretação complexa, utilizando outras técnicas de agrupamento, como hierárquicos e dispersão no plano.

Conforme o Quadro 6, os altos valores de dissimilaridade e o grande número de grupos e subgrupos formados sugerem alta diversidade genética entre os genótipos, sendo a maior e a menor estimativa de divergência genética obtida entre todos os acessos de 5,08 e 0,01. Considerando só os genótipos de $E$. urohylla, a maior e menor estimativa foi 4,81 e 0,01, respectivamente; considerando só os genótipos de E. grandis, de 4,01 e 0,04. Não parece haver diferença significativa entre as médias das estimativas de divergência obtidas das espécies E. urohylla e E. grandis, pelo teste " $t$ " a $1 \%$ de probabilidade. A tendência de agrupamento observada entre os genótipos da mesma espécie, associada aos altos valores de diversidade genética obtidos entre espécies, indica que estratégias de melhoramento com manutenção da identidade genética das populações vêem sendo eficientes e que o programa vem mantendo uma variabilidade genética satisfatória ao longo dos ciclos de seleção.

\subsection{Desempenho das progênies híbridas}

Utilizando-se os dados estimados nos testes de progênies de E. urophylla e de E. grandis, foi feita uma simulação do comportamento das progênies híbridas, com relação ao ganho em DAP (Quadro 7). Optou-se pela escolha dos pares de genitores para cruzamentos considerando as maiores distâncias genéticas entre o primeiro indivíduo selecionado de E. urophylla e os três primeiros selecionados de E. grandis, que se caracterizaram pelas maiores estimativas de distância genética. Para a definição dos cruzamentos em dialelo parcial circulante envolvendo 100 genitores de cada espécie, adotou-se um esquema em que cada genitor foi cruzado com três outros que apresentaram maiores estimativas de distância genéticas.

As estimativas de ganhos genéticos em DAP foram similares pelo uso da herdabilidade média $(35,68 \%)$ e pelo uso da divergência genética $(38,50 \%)$, conforme o Quadro 7. Os cruzamentos interespecíficos entre os indivíduos mais divergentes têm sido utilizados em trabalhos recentes (SCAPIM et al., 1994; MURO ABAD et al., 2005), e neste trabalho essa estratégia foi utilizada inserida em um procedimento de seleção que visa reunir os pontos fortes de metodologias promissoras do melhoramento florestal, a acurácia na precisão das estimativas dada pelo procedimento REML/BLUP, associada à informação de divergência genética, visando obter as melhores combinações para a obtenção dos híbridos.

\section{CONCLUSÕES}

- O procedimento REML mostra-se adequado para estimativas de parâmetros genéticos em E. urophylla e E. grandis, com alta confiabilidade, conforme evidenciado pela acurácia.

- Os altos ganhos preditos decorreram da alta variabilidade genética presente nas populações, evidenciadas pelas análises de variâncias e pelos coeficientes de herdabilidade.

- Os desempenhos das progênies híbridas mostramse favoráveis à realização de cruzamentos controlados em dialelo parcial circulante, com ganhos da ordem de $35 \%$.

- Os genitores selecionados apresentam alta divergência genética, com uma clara tendência de agrupamento entre as espécies E. grandis e E. urophylla e os híbridos interespecíficos.

R. Árvore, Viçosa-MG, v.31, n.6, p.977-987, 2007 
Quadro 7 - Valor genotípico do híbrido e ganho genético do cruzamento dos 10 primeiros indivíduos de Eucalyptus urophylla $x$ Eucalyptus grandis de maior efeito genético aditivo e com maior distância genética entre eles, pelo uso da heterose média e da divergência genética

Table 7 - Genetic hybrid value and genetic gain from breeding 10 E. urophylla $x$ E. grandis genotypes with the highest additive values and genetic divergence

\begin{tabular}{|c|c|c|c|c|c|c|}
\hline \multirow[t]{2}{*}{ E. urophylla } & \multirow[t]{2}{*}{ E. grandis } & \multirow[t]{2}{*}{ Dist. Gen. } & \multicolumn{2}{|c|}{ Heterose média } & \multicolumn{2}{|c|}{ Divergência Genética } \\
\hline & & & $\hat{G}_{u g}$ & $\hat{G}_{s}(\%)$ & $\hat{G}_{u g}$ & $\hat{G}_{s}(\%)$ \\
\hline \multirow{4}{*}{101} & 4 & 3,5007 & 16,21 & 36,82 & 17,93 & 51,38 \\
\hline & 5 & 3,4984 & 16,21 & 36,82 & 17,93 & 51,36 \\
\hline & 9 & 3,5096 & 16,19 & 36,70 & 17,93 & 51,33 \\
\hline & 5 & 2,7592 & 16,15 & 36,32 & 17,13 & 44,61 \\
\hline \multirow[t]{3}{*}{102} & 9 & 2,7750 & 16,13 & 36,29 & 17,13 & 44,62 \\
\hline & 10 & 2,8043 & 16,13 & 36,15 & 17,15 & 44,83 \\
\hline & 2 & 2,4249 & 16,29 & 37,50 & 16,94 & 42,97 \\
\hline \multirow[t]{3}{*}{103} & 4 & 2,2126 & 16,09 & 35,81 & 16,52 & 39,49 \\
\hline & 6 & 2,5640 & 16,08 & 35,73 & 16,86 & 33,93 \\
\hline & 1 & 2,2644 & 16,24 & 37,12 & 16,73 & 41,24 \\
\hline \multirow[t]{3}{*}{104} & 7 & 2,3916 & 16,01 & 35,28 & 16,63 & 40,37 \\
\hline & 10 & 2,4109 & 16,00 & 35,10 & 16,64 & 40,45 \\
\hline & 1 & 2,1506 & 16,23 & 37,03 & 16,47 & 39,05 \\
\hline \multirow[t]{3}{*}{105} & 2 & 2,0152 & 16,21 & 36,90 & 16,45 & 38,88 \\
\hline & 8 & 1,5822 & 16,00 & 35,10 & 16,81 & 33,45 \\
\hline & 4 & 4,4422 & 16,00 & 35,09 & 16,67 & 57,60 \\
\hline \multirow[t]{3}{*}{106} & 5 & 4,3540 & 16,00 & 35,09 & 16,58 & 56,85 \\
\hline & 9 & 4,4947 & 15,99 & 35,00 & 18,71 & 57,91 \\
\hline & 1 & 0,9555 & 16,18 & 36,61 & 15,36 & 29,68 \\
\hline \multirow[t]{3}{*}{107} & 3 & 0,6111 & 15,98 & 34,92 & 14,82 & 25,08 \\
\hline & 7 & 1,0941 & 15,97 & 34,84 & 15,29 & 29,08 \\
\hline & 2 & 1,3864 & 16,18 & 36,60 & 15,79 & 33,28 \\
\hline \multirow[t]{3}{*}{108} & 3 & 1,0015 & 16,00 & 35,10 & 15,23 & 28,55 \\
\hline & 7 & 1,5398 & 15,97 & 34,80 & 15,73 & 32,80 \\
\hline & 6 & 0,8436 & 15,96 & 34,71 & 14,71 & 24,17 \\
\hline \multirow[t]{3}{*}{109} & 3 & 0,5277 & 15,99 & 35,01 & 15,06 & 27,13 \\
\hline & 8 & 0,7336 & 15,96 & 34,71 & 14,91 & 25,91 \\
\hline & 6 & 1,5197 & 15,94 & 34,54 & 15,68 & 32,37 \\
\hline \multirow[t]{2}{*}{110} & 8 & 0,9775 & 15,94 & 34,54 & 15,14 & 27,80 \\
\hline & 10 & 1,1191 & 15,93 & 34,46 & 15,27 & 28,91 \\
\hline Média & & & & 35,68 & & 38,50 \\
\hline
\end{tabular}

\section{5- REFERÊNCIAS}

CRUZ, C. D. Programa GENES - aplicativo computacional em genética e estatística. Manual do usuário. Viçosa, MG: Universidade Federal de Viçosa, 1997. 442p.

CRUZ, C. D.; PIRES, I. E. Curso sobre capacidade combinatória de genótipos de eucalipto. Viçosa, MG: Universidade Federal de Viçosa/Sociedade de Investigação Florestal, 1996. 30p.

CRUZ, C. D.; REGAZZI, A. J. Modelos

biométricos aplicados ao melhoramento genético. Viçosa, MG:

Universidade Federal de Viçosa, 1994. 390p.

R. Árvore, Viçosa-MG, v.31, n.6, p.977-987, 2007
CRUZ, C. D.; CARNEIRO, P. C. S. Modelos

biométricos aplicados ao melhoramento genético. Viçosa, MG:

Universidade Federal de Viçosa, 2003. v.2. 585p.

FALCONER, D. S. Introdução à genética quantitativa. Tradução por M.A. Silva; J.C. Silva. Viçosa, MG: Universidade Federal de Viçosa, Imprensa Universitária, 1987. 279p.

GARCIA, C. H. Tabelas para classificação do coeficiente de variação. Piracicaba: IPEF, 1989. 11p. (Circular Técnica, 171). 
GARCIA, C. H.; NOGUEIRA, M. C. S. N. Utilização da metodologia REML/BLUP na seleção de clones de eucalipto. Scientia Forestalis, n.68, p.107-112, 2005.

HENDERSON, C. R.; KEMPTHORNE, O.; SEARLE, S. R.; KROSIGK VON, C. M. The estimation of environmental and genetic trends from records subject to culling. Biometrics, v.15, n.6, p.192-218, 1959.

KEMPHORNE, O.; CURNOW, R. N. The partial diallel cross. Biometrics, v.17, p.229-250, 1961.

MARTINS, I. S. et al. Eficiência da seleção direta e indireta e índices de seleção em Eucalyptus grandis. Revista Árvore, v.27, n.3, p.327-333, 2003.

MORAES, M. L. T. Variação genética da densidade básica da madeira em progênies de Eucalyptus grandis Hill ex Maiden e suas relações com as características de crescimento. 1987. $115 \mathrm{f}$. Dissertação (Mestrado em Ciências Florestais) Escola Superior de Agricultura Luiz de Queiroz, Piracicaba, 1987.

MURO ABAD, J. I. et al. Crosses recommendation method for obtaining Eucalyptus spp. hybrids assisted by molecular markers. Crop Breeding and Applied Biotechnology, v.5, p.71-73, 2005.

PIRES, I. E. Eficiência da seleção combinada no melhoramento genético de Eucalyptus spp. 1996. 116f . Tese (Doutorado em Genética e Melhoramento) Universidade Federal de Viçosa, Viçosa, MG, 1996.

RESENDE, M. D. V. et al. Acurácia seletiva, intervalos de confiança e variâncias de ganhos genéticos associados a 22 métodos de seleção em Pinus caribaea var. hondurensis. Boletim de Pesquisa Florestal, n. 21, p.1-18, 1990.

RESENDE, M. D. V.; HIGA, A. R. Estratégias de melhoramento para eucaliptos visando a seleção de híbridos. Boletim de Pesquisa

Florestal, n. 21, p.49-60, 1990.

RESENDE, M. D. V. et al. Efeito do tamanho amostral na estimativa da herdabilidade em espécies perenes. Floresta, v.28, p.51-63, 1998.
RESENDE, M. D. V. Análise estatística de modelos mistos via REML/BLUP na experimentação em melhoramento de plantas perenes. Colombo: Embrapa Florestas, 2000. 101p. (Documentos, 47).

RESENDE, M. D. V. SELEGEN-REML/BLUP Seleção genética computadorizada: manual do usuário. Colombo: Embrapa-CNPF, 2002a. 67p.

RESENDE, M. D. V. Genética biométrica e estatística no melhoramento de plantas perenes. Brasília: Embrapa Informação e Tecnológica, 2002b. 975p.

RESENDE, M. D.V.; BERTOLUCCI, F. L. G. Maximization of genetic gain with restriction on effective population size and inbreeding in Eucalyptus grandis. In: IUFRO CONFERNCE "EUCALYPT PLANTATIONS: IMPROVING FIBRE YIELD AND QUALITY”, Hobart, 1995. Proceedings... Hobart: CRC for Temperate Hardwood Forestry, 1995. p.167-170.

RESENDE, R. M. S. Avaliação genética de populações e progênies de erva-mate (Ilex paraguariensis Saint Hilaire) em três locais no estado do Paraná. 2001. 156f. Tese (Doutorado em Genética) Universidade Federal do Paraná, Curitiba, 2001.

SAMPAIO P. T. B.; RESENDE M. D. V.; ARAÚJO A. J. Estimativas de parâmetros genéticos e métodos de seleção para o melhoramento genético de Pinus oocarpa Schiede. Pesquisa Agropecuária Brasileira, v.37, n.5, p.625-636, 2002.

SCAPIM, C.A.; CRUZ, C. D.; PIRES, I. E. Estudo da diversidade genética em Eucalyptus camaldulensis. In: WORKSHOP - Métodos de Seleção, LOCAL, 1994. Anais... LOCAL: Comissão Técnica de Genética e Melhoramento, 1994. p.45-57.

VAN VLECK, L. D.; POLLAK, E. J.; OLTENACU, E. A. B. Genetics for the animal sciences. New York: W. H. Freemam, 1987. 391p.

R. Árvore, Viçosa-MG, v.31, n.6, p.977-987, 2007 
